\title{
TEXT SEGMENTATION BASED ON SIMILARITY BETWEEN WORDS
}

\author{
Hideki Kozima \\ Course in Computer Science and Information Mathematics, \\ Graduate School, University of Electro-Communications \\ 1-5-1, Chofugaoka, Chofu, \\ Tokyo 182, Japan \\ (xkozima@phaeton.cs.uec.ac.jp)
}

\begin{abstract}
This paper proposes a new indicator of text structure, called the lexical cohesion profile (LCP), which locates segment boundaries in a text. A text segment is a coherent scene; the words in a segment are linked together via lexical cohesion relations. LCP records mutual similarity of words in a sequence of text. The similarity of words, which represents their cohesiveness, is computed using a semantic network. Comparison with the text segments marked by a number of subjects shows that LCP closely correlates with the human judgments. LCP may provide valuable information for resolving anaphora and ellipsis.
\end{abstract}

\section{INTRODUCTION}

A text is not just a sequence of words, but it has coherent structure. The meaning of each word can not be determined until it is placed in the structure of the text. Recognizing the structure of text is an essential task in text understanding, especially in resolving anaphora and ellipsis.

One of the constituents of the text structure is a text segment. A text segment, whether or not it is explicitly marked, as are sentences and paragraphs, is defined as a sequence of clauses or sentences that display local coherence. It resembles a scene in a movie, which describes the same objects in the same situation.

This paper proposes an indicator, called the lexical cohesion profile (LCP), which locates segment boundaries in a narrative text. LCP is a record of lexical cohesiveness of words in a sequence of text. Lexical cohesiveness is defined as word similarity (Kozima and Furugori, 1993) computed by spreading activation on a semantic network. Hills and valleys of LCP closely correlate with changing of segments.

\section{SEGMENTS AND COHERENCE}

Several methods to capture segment boundaries have been proposed in the studies of text structure. For example, cue phrases play an impor- tant role in signaling segment changes. (Grosz and Sidner, 1986) However, such clues are not directly based on coherence which forms the clauses or sentences into a segment.

Youmans (1991) proposed VMP (vocabulary management profile) as an indicator of segment boundaries. VMP is a record of the number of new vocabulary terms introduced in an interval of text. However, VMP does not work well on a high-density text. The reason is that coherence of a segment should be determined not only by reiteration of words but also by lexical cohesion.

Morris and Hirst (1991) used Roget's thesaurus to determine whether or not two words have lexical cohesion. Their method can capture almost all the types of lexical cohesion, e.g. systematic and non-systematic semantic relation. However it does not deal with strength of cohesiveness which suggests the degree of contribution to coherence of the segment.

\section{Computing Lexical Cohesion}

Kozima and Furugori (1993) defined lexical cohesiveness as semantic similarity between words, and proposed a method for measuring it. Similarity between words is computed by spreading activation on a semantic network which is systematically constructed from an English dictionary (LDOCE).

The similarity $\sigma\left(w, w^{\prime}\right) \in[0,1]$ between words $w, w^{\prime}$ is computed in the following way: (1) produce an activated pattern by activating the node $w ;(2)$ observe activity of the node $w^{\prime}$ in the activated pattern. The following examples suggest the feature of the similarity $\sigma$ :

$$
\begin{array}{lll}
\sigma \text { (cat, } & \text { pet }) & =0.133722, \\
\sigma \text { (cat, } & \text { hat }) & 0.001784, \\
\sigma \text { (waiter, } & \text { restaurant }) & =0.175699, \\
\sigma \text { (painter, } & \text { restaurant }) & =0.006260 .
\end{array}
$$

The similarity $\sigma$ depends on the significance $s(w) \in[0,1]$, i.e. normalized information of the word $u$ in West's corpus (1953). For example:

$$
\mathrm{s}(\mathrm{red})=0.500955, \mathrm{~s}(\mathrm{and})=0.254294 \text {. }
$$




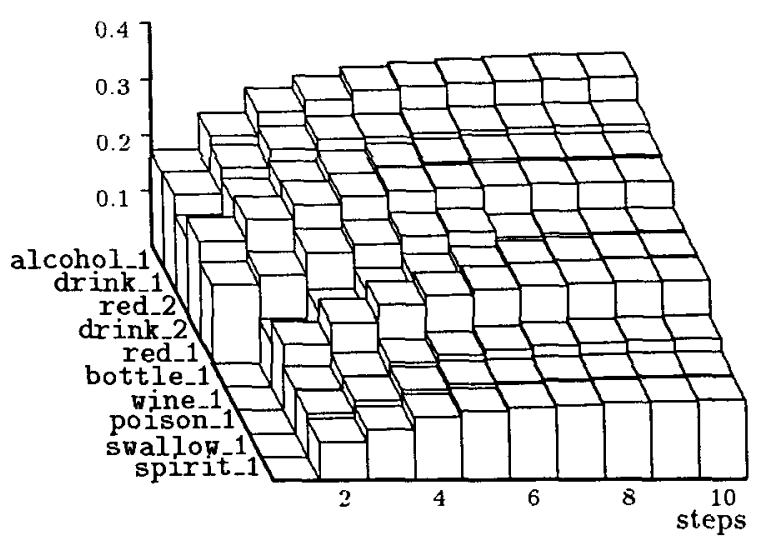

Figure 1. An activated pattern of a word list (produced from \{red, alcoholic, drink\}).

The following examples show the relationship between the word significance and the similarity:

$$
\begin{array}{lll}
\sigma \text { (waiter, } & \text { waiter }) & =0.596803, \\
\sigma \text { (red, } & \text { blood }) & =0.111443, \\
\sigma \text { (of, } & \text { blood }) & =0.001041 .
\end{array}
$$

\section{LEXICAL COHESION PROFILE}

LCP of the text $T=\left\{w_{1}, \cdots, w_{N}\right\}$ is a sequence $\left\{c\left(S_{1}\right), \cdots, c\left(S_{N}\right)\right\}$ of lexical cohesiveness $c\left(S_{i}\right) . S_{i}$ is the word list which can be seen through a fixedwidth window centered on the $i$-th word of $T$ :

$$
\begin{array}{cl}
S_{i}=\left\{w_{l}, w_{l+1}, \cdots, w_{i-1}, w_{i}, w_{i+1}, \cdots, w_{r-1}, w_{r}\right\}, \\
l=i-\Delta \quad
\end{array}
$$

LCP treats the text $T$ as a word list without any punctuation or paragraph boundaries.

\section{Cohesiveness of a Word List}

Lexical cohesiveness $c\left(S_{i}\right)$ of the word list $S_{i}$ is defined as follows:

$$
c\left(S_{i}\right)=\sum_{w \in S_{i}} s(w) \cdot a\left(P\left(S_{i}\right), w\right),
$$

where $a\left(P\left(S_{i}\right), w\right)$ is the activity value of the node $w$ in the activated pattern $P\left(S_{i}\right)$. $P\left(S_{i}\right)$ is produced by activating each node $w \in S_{i}$ with strength $s(w)^{2} / \sum s(w)$. Figure 1 shows a sample pattern of $\{$ red, alcoholic, drink $\}$. (Note that it has highly activated nodes like bottle and wine.)

The definition of $c\left(S_{i}\right)$ above expresses that $c\left(S_{i}\right)$ represents semantic homogeneity of $S_{i}$, since $P\left(S_{i}\right)$ represents the average meaning of $w \in S_{i}$. For example:

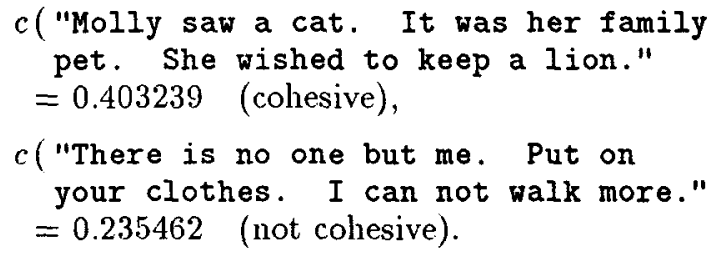

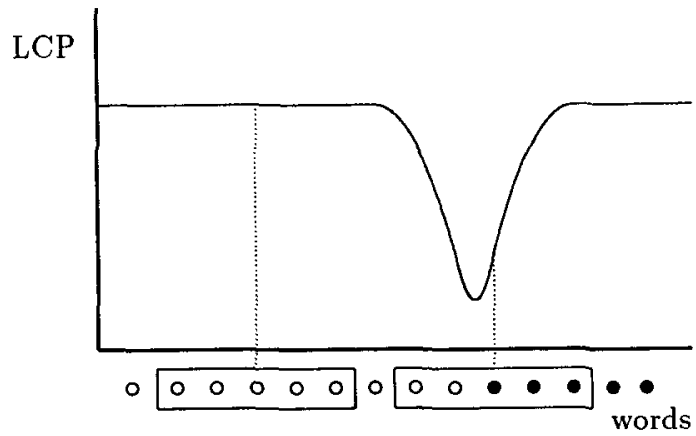

Figure 2. Correlation between LCP and text segments.

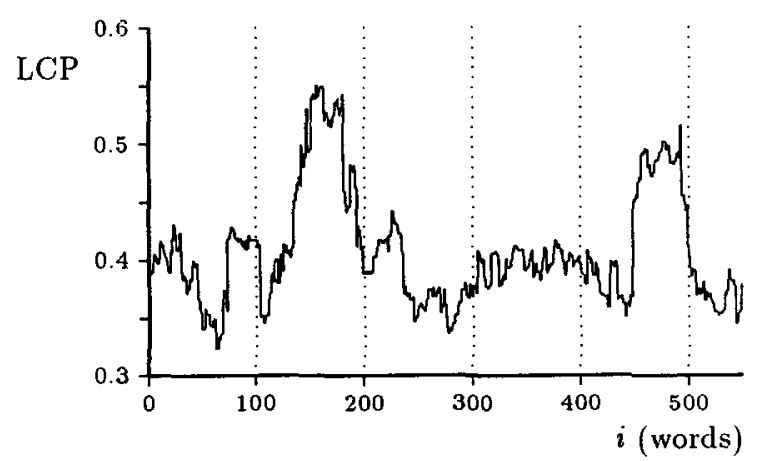

Figure 3. An example of LCP

(using rectangular window of $\Delta=25$ )

\section{LCP and Its Feature}

A graph of LCP, which plots $c\left(S_{i}\right)$ at the text position $i$, indicates changing of segments:

- If $S_{i}$ is inside a segment, it tends to be cohesive and makes $c\left(S_{i}\right)$ high.

- If $S_{i}$ is crossing a segment boundary, it tends to semantically vary and makes $c\left(S_{i}\right)$ low.

As shown in Figure 2, the segment boundaries can be detected by the valleys (minimum points) of LCP.

The LCP, shown in Figure 3, has large hills and valleys, and also meaningless noise. The graph is so complicated that one can not easily determine which valley should be considered as a segment boundary.

The shape of the window, which defines weight of words in it for pattern production, makes LCP smooth. Experiments on several window shapes (e.g. triangle window, etc.) shows that Hanning window is best for clarifying the macroscopic features of LCP.

The width of the window also has effect on the macroscopic features of LCP, especially on separability of segments. Experiments on several window widths $(\Delta=5 \sim 60)$ reveals that the Hanning window of $\Delta=25$ gives the best correlation between LCP and segments. 


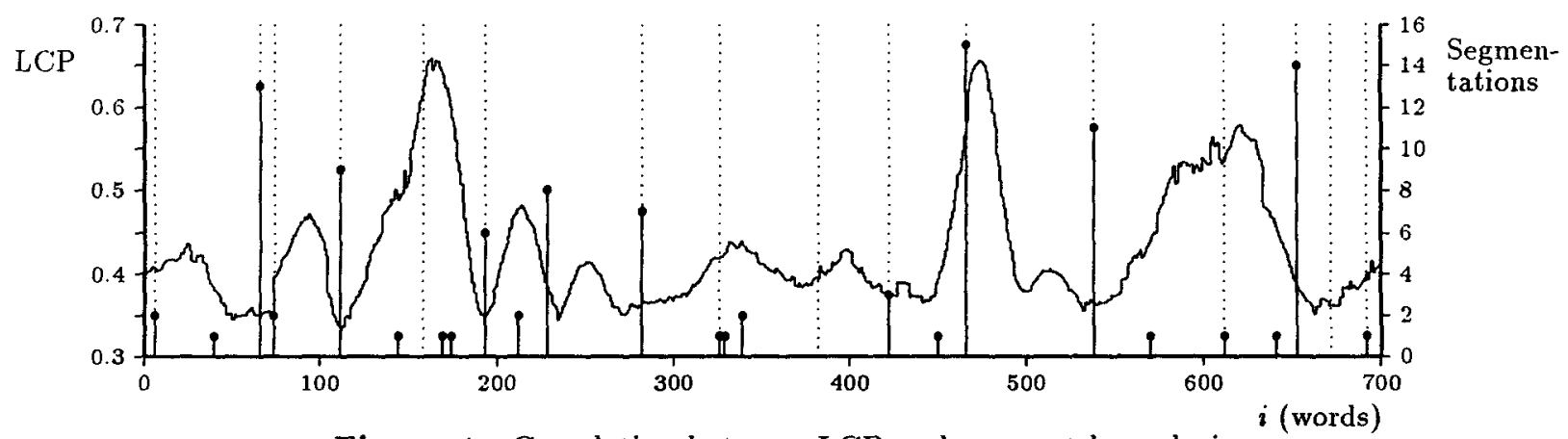

Figure 4. Correlation between LCP and segment boundaries.

\section{VERIFICATION OF LCP}

This section inspects the correlation between LCP and segment boundaries perceived by the human judgments. The curve of Figure 4 shows the LCP of the simplified version of O.Henry's "Springtime à la Carte" (Thornley, 1960). The solid bars represent the histogram of segment boundaries reported by 16 subjects who read the text without paragraph structure.

It is clear that the valleys of the LCP correspond mostly to the dominant segment boundaries. For example, the clear valley at $i=110$ exactly corresponds to the dominant segment boundary (and also to the paragraph boundary shown as a dotted line).

Note that LCP can detect segment changing of a text regardless of its paragraph structure. For example, $i=156$ is a paragraph boundary, but neither a valley of the LCP nor a segment boundary; $i=236$ is both a segment boundary and approximately a valley of the LCP, but not a paragraph boundary.

However, some valleys of the LCP do not exactly correspond to segment boundaries. For example, the valley near $i=450$ disagree with the segment boundary at $i=465$. The reason is that lexical cohesion can not cover all aspect of colherence of a segment; an incoherent piece of text can be lexically cohesive.

\section{CONCLUSION}

This paper proposed LCP, an indicator of segment changing, which concentrates on lexical cohesion of a text segment. The experiment proved that LCP closely correlate with the segment boundaries captured by the human judgments, and that lexical cohesion plays main role in forming a sequence of words into segments.

Text segmentation described here provides basic information for text understanding:

- Resolving anaphora and ellipsis:

Segment boundaries provide valuable restriction for determination of the referents.
- Analyzing text structure:

Segment boundaries can be considered as segment switching (push and pop) in hierarchical structure of text.

The segmentation can be applied also to text summarizing. (Consider a list of average meaning of segments.)

In future research, the author needs to examine validity of LCP for other genres - Hearst (1993) segments expository texts. Incorporating other clues (e.g. cue phrases, tense and aspect, etc.) is also needed to make this segmentation method more robust.

\section{ACKNOWLEDGMENTS}

The author is very grateful to Dr. Teiji Furugori, University of Electro-Communications, for his insightful suggestions and comments on this work.

\section{REFERENCES}

Grosz, Barbara J., and Sidner, Candance L. (1986). "Attention, intentions, and the structure of discourse." Computational Linguistics, 12, 175-204.

Halliday, Michael A. K., Hasan, Ruqaiya (1976). Chesion in English. Longman.

Hearst, Marti, and Plaunt, Christian (1993). "Subtopic structuring for full-length document access," to appear in SIGIR 1993, Pittsburgh, PA.

Kozima, Hideki, and Furugori, Teiji (1993). "Similarity between words computed by spreading activation on an English dictionary." to appear in Proceedings of EACL-93.

Morris, Jane, and Hirst, Graeme (1991). "Lexical cohesion computed by thesaural relations as an indicator of the structure of text." Computational Linguistics, 17, 21-48.

Thornley, G. C. editor (1960). British and American Short Stories, (Longman Simplified English Series). Longman.

West, Michael (1953). A General Service List of English Words. Longman.

Youmans, Gilbert (1991). "A new tool for discourse analysis: The vocabulary-management profile." Language, 67, 763-789. 